\title{
Determination of Base Flipping Free Energy Landscapes from Nonequilibrium Stratification
}

\author{
Xiaohui Wang ${ }^{1,2}$ and Zhaoxi Sun ${ }^{1,3^{*}}$ \\ ${ }^{1}$ State Key Laboratory of Precision Spectroscopy, School of Chemistry and Molecular Engineering, East China \\ Normal University, Shanghai 200062, China \\ ${ }^{2}$ Institute of Computational Science, Università della Svizzera italiana (USI), Via Giuseppe Buffi 13, CH-6900, \\ Lugano, Ticino, Switzerland \\ ${ }^{3}$ Computational Biomedicine (IAS-5/INM-9), Forschungszentrum Jülich, Jülich 52425, Germany
}

*To whom correspondence should be addressed: z.sun@fz-juelich.de

\begin{abstract}
Correct calculation of the variation of free energy upon base flipping is crucial in understanding the dynamics of DNA systems. The free energy landscape along the flipping pathway gives the thermodynamic stability and the flexibility of base-paired states. Although numerous free energy simulations are performed in the base flipping cases, no theoretically rigorous nonequilibrium techniques are devised and employed to investigate the thermodynamics of base flipping. In the current work, we report a general nonequilibrium stratification scheme for efficient calculation of the free energy landscape of base flipping in DNA duplex. We carefully monitor the convergence behavior of the equilibrium sampling based free energy simulation and the nonequilibrium stratification and determine the empirical length of time blocks required for converged sampling. Comparison between the performances of equilibrium umbrella sampling and nonequilibrium stratification is given. The results show that nonequilibrium free energy simulation is able to give similar accuracy and efficiency compared with the equilibrium enhanced sampling technique in the base flipping cases. We further test a convergence criterion we previously proposed and it comes out that the convergence behavior determined by this criterion agrees with those given by the time-invariant behavior of PMF and the nonlinear dependence of standard deviation on the sample size.
\end{abstract}




\section{Introduction}

Understanding the fundamental driving force of conformational changes in DNA systems are of great importance due to the unique functionality of DNA molecules. Duplex, triplex and various other conformations are observed in DNA systems. ${ }^{1-5}$ The information carried by DNA systems is mostly buried in the hydrophobic core of the helix and the phenomenon called base flipping enables the interaction between external agents and the bases. Base flipping is widely observed in DNA methylation, ${ }^{6-9}$ melting, ${ }^{10-16}$ bubbling, ${ }^{17-18}$ breathing, ${ }^{19-21}$ and protein-DNA interaction. ${ }^{22}$ The experimental community remains very active on base flipping. ${ }^{23}$ For instance, the protonation-dependent base flipping is observed in RNA systems recently. ${ }^{24}$ The protonation-induced conformational change includes the transformation from unpaired GA mismatch to paired G-HA ${ }^{+}$base pair. Recent experimental techniques such as hydrogen exchange (HX) experiments with UV spectrum or nuclear magnetic resonance (NMR) for detection ${ }^{25}$ and formaldehyde kinetics ${ }^{15}$ are widely employed. From the experimental data, the major features of the physical systems are extracted and theoretical models are devised. In the early stage of theoretical study, the models are mostly extended Ising models. The double rod-like model is one of the extended Ising models proposed. ${ }^{26-27}$ The helix-coil transition model originally applied to helix formation in protein folding ${ }^{16,28}$ is altered to study the thermodynamics of DNA duplex. ${ }^{15-16,}$ 29 The Peyrad-Bishop-Dauxois (PBD) mesoscopic model uses the hydrogen bond interactions and the stacking interactions between neighboring bases to describe the potential energy function and other factors such as the DNA-solvent interactions are included implicitly in the two terms in parameterization. ${ }^{30-32}$ The PBD model is employed for simulations of DNA systems and agreements between predictions and experimental data are observed. ${ }^{33-36}$ These models rely on simplified description of interactions and thus have low transferability. The development of computer architecture, efficient algorithms and optimized software enable simulations of DNA molecules at atomic details. Using all-atom force field to describe the system, ${ }^{37-41}$ with enhanced sampling techniques to increase the sampling efficiency, ${ }^{42-48}$ we can perform ns, $\mu \mathrm{s}, \mathrm{ms}$ and even longer molecular dynamics (MD) simulations with supercomputers. Examples of recent reports of nucleotides simulations are the protonation-dependent base flipping ${ }^{49}$ polarization induced variation in the free energy barrier in DNA base flipping,${ }^{50}$ and the ion induced BI<->BII conformational transition. ${ }^{51}$ Recent benchmarks indicate that modern computer simulations already have some predictive power for DNA systems. ${ }^{52-54}$

Free energy profiles or free energy landscapes along the base flipping pathway give the thermodynamic tendency of the transitions between the base-paired and flipping-out states. Numerous reports on base flipping rely on free energy landscapes to investigate the mechanism and mutation-dependent behavior of base flipping..$^{49-50,55-67}$ Enhanced sampling techniques are employed to enhance the sampling efficiency and reweighting schemes are used to recover the unbiased statistics. The post-processing estimators are mostly based on free energy perturbation (FEP) ${ }^{68}$. For 
instance, the weighted histogram analysis method (WHAM) $)^{69-70}$ is the multistate version of FEP. Another perturbation-based estimator is the variational free energy profile (vFEP), ${ }^{71}$ which relies on maximum likelihood estimation and cubic spline interpolation. In most studies, equilibrium sampling based techniques are employed to study base flipping and examples are replica exchange variants, accelerated MD, umbrella sampling, and the quasi-equilibrium metadynamics. ${ }^{49-50,56,59,72-73}$ By contrast, the nonequilibrium work based enhanced sampling technique, the steered MD (SMD) approach, is seldom used to investigate the thermodynamics of base flipping. In some studies, the SMD approach is used to investigate the feasibility and the behavior of base pairs upon pulling in the nonequilibrium ensemble, but no equilibrium thermodynamic information is recovered. ${ }^{74-76}$ In others, the SMD approach is coupled with the nonequilibrium generalizations of FEP, termed as Jarzynski's Identity (JI) ${ }^{77}$ and Crooks' Equation (CE) ${ }^{78}$ to compute the free energy difference between the base-paired state and the flipping-out state. ${ }^{79-80}$ However, the extreme length of pulling distance results in huge dissipation and slow convergence. Also, previous nonequilibrium construction of free energy profiles of base flipping does not define the computational cost for each independent sample in a theoretically rigorous way ${ }^{80}$ thus further lowering the performance of nonequilibrium pulling. The above theoretical and technical difficulties hinder the applicability of nonequilibrium free energy simulation on base flipping. To fully exploit the applicability and maximize the performance of nonequilibrium techniques for investigation of the base flipping processes, we proposed a general nonequilibrium stratification scheme for feasible construction of equilibrium free energy landscapes from multi-stage nonequilibrium pulling simulations. The whole base flipping process is divided into a series of short segments and nonequilibrium pulling simulations are performed between them to accumulate the microscopic nonequilibrium work. These microscopic nonequilibrium works are ultimately combined with the statistically optimal and asymptotically unbiased bidirectional reweighting estimator of CE to recover the unbiased free energy differences along the base flipping pathway, giving the free energy landscape. We compare the performance of the nonequilibrium stratification scheme with that of the equilibrium umbrella sampling coupled with WHAM $^{69-70}$ and vFEP. $^{71}$ The convergence of equilibrium umbrella sampling and nonequilibrium pulling simulations is monitored with care, and the empirical simulation time required for convergence of these methods is determined. The results show that the nonequilibrium scheme is able to give comparable accuracy and efficiency with the equilibrium sampling technique.

\section{Methodology}

The SMD approach and the construction of potential of mean force (PMF) from nonequilibrium works. During conformational changes of physical systems, there are often several slow degrees of freedom important for describing the process. These slow degrees of freedom are often called as collective variable (CV), order parameter or 
reaction coordinate. The SMD method biases the simulation by driving the system along the CV space with a pre-defined time-dependent harmonic potential according to the schedule $\xi_{0} .{ }^{81}$ The harmonic potential is defined as

$$
h(\mathbf{q})=\frac{k}{2}\left(\xi(\mathbf{q})-\xi_{0}(t)\right)^{2}
$$

, where $h$ is the harmonic potential, $\mathbf{q}$ represents the coordinate vector, $k$ denotes the force constant of the harmonic potential, $\xi_{0}$ represents the time-dependent $\mathrm{CV}$ defining the configurational pathway, and $\xi$ refers to the current value of $\mathrm{CV}$. As the harmonic restraint cannot fix the value of $\mathrm{CV}$ to a specific point, often the non-fixed behavior needs to be considered in nonequilibrium reweighting. However, in the stiff spring limit, the distribution of $\mathrm{CV}$ under restraints is extremely narrow and the need to add such a correction vanishes. ${ }^{82-84}$

The test system is the model poly $\mathrm{A}_{7}-\mathrm{T}_{7}$ DNA duplex and the flipping base is the middle A4 group. The slow structural observable used to investigate the base flipping process is the pseudo-dihedral defined by four centers of mass (COM). As is shown at the center of Fig.1, the pseudo-dihedral is defined by the flipping base of A4, the sugar moiety of A4, the sugar moiety of A5 (the 3' side of A4) and the base pair of A5-T10. An illustration of the base flipping process is also given in Fig.1.

As the whole pulling process from $0^{\circ}$ to $360^{\circ}$ is quite long for a single run, the pulling speed must be extremely slow to reduce dissipation and narrow the distribution of nonequilibrium work. Therefore, to reduce dissipation the staging regime, the stratification of the nonequilibrium pulling, should be applied. The whole process is divided into a series of small segments and the nonequilibrium pulling are performed between neighboring states. As the stiff string limit is achieved, we define the conformational state by the value of CV with $2^{\circ}$ increments. Such setting in dihedral pulling is employed in our previous works and satisfactory results can be obtained. ${ }^{85-86}$ As a result, there are 180 conformational states in total, as is shown in Fig. 1. According to the closure of the thermodynamic cycle shown in Fig. 1, the conformational states at $0^{\circ}$ and $360^{\circ}$ are exactly the same and thus their free energies are equal.

In Fig. 1, bidirectional arrows are used to represent both the bidirectional pulling between neighboring conformational states ( $\xi$ increasing and $\xi$ decreasing) and the bidirectional reweighting procedure used to recover the unbiased free energy differences. The dimensionless free energy difference between two neighboring states is obtained from the asymptotically unbiased and statistically optimal estimator of CE defined in Eq. (2) and the corresponding variance is defined in Eq. (3), ${ }^{78,87-88}$ 


$$
\begin{gathered}
\left\{\begin{array}{c}
\Delta A_{i j}=\ln \frac{\left\langle f\left(W_{j i}+C\right)\right\rangle_{j}}{\left\langle f\left(W_{i j}-C\right)\right\rangle_{i}}+C \\
C=\Delta A_{i j}+\ln \left(\frac{n_{j}}{n_{i}}\right)
\end{array}\right. \\
\sigma_{i j}^{2}=\frac{\operatorname{Var}\left(f_{i j}\right)}{n_{i}^{2} f_{i j}^{2}}+\frac{\operatorname{Var}\left(f_{j i}\right)}{n_{j}^{2} f_{j i}^{2}}
\end{gathered}
$$

, where the canonical bracket with subscript $i$ refers to canonical average over nonequilibrium realizations initiated from state $i, W_{i j}$ denotes the dimensionless work accumulated during nonequilibrium pulling initiated from state $i$ and ended in state $j, n$ is the sample size in each ensemble, $f$ is the Fermi function, $\Delta A_{i j}=A_{j}-A_{i}$ represents the dimensionless free energy difference and Var denotes the absolute variance. The definition of $f_{i j}$ is

$$
\begin{aligned}
& f_{i j}=\left\langle f\left(W_{i j}+C_{i j}\right)\right\rangle_{i} \\
& f_{j i}=\left\langle f\left(W_{j i}-C_{i j}\right)\right\rangle_{j} \\
& n_{i j} f_{i j}=n_{j i} f_{j i} \text { from SCF of CE } \\
& j=i+1
\end{aligned}
$$

As nonequilibrium reweighting estimators require the initial configurations to be extracted from equilibrium ensembles, short initial configurational sampling in the equilibrium ensemble is needed. As the free energy estimator requires statistically independent samples as input data, the autocorrelation time of the CV (the flipping dihedral) in each conformational state $\tau_{i}$ is computed and independent configurations are subsampled from the initial dataset by the statistical inefficiency $\phi_{e q, i}=1+2 \tau_{i}$. Therefore, in the initial configurational sampling at state $i$, each independent configuration requires the sampling time of $\phi_{e q, i}$. Each nonequilibrium pulling lasts for $\tau_{\mathrm{NEW}}$. Thus each bidirectional nonequilibrium pulling lasts for $\phi_{\mathrm{NEW}, i}=2 \tau_{\mathrm{NEW}}$. Combine the above quantities we obtain the overall computational cost for each pulling as

$$
\phi_{i}=\phi_{\mathrm{NEW}, i}+\phi_{e q, i}
$$

. As the free energy landscape only depicts the variation of free energy along CVs, only the free energy differences between different states are meaningful. Therefore, we can define a reference state with a free energy of zero. Here we set the conformational state at $0^{\circ}$ as the reference state 1 . The free energies of the other states (e.g. state $k$ ) can be obtained by accumulating the free energy differences of multiple segments, namely 


$$
\begin{aligned}
& A_{k}=\Delta A_{1 k}=\sum_{i=1}^{k-1} \Delta A_{i, i+1} \\
& \sigma_{1 k}^{2}=\sum_{i=1}^{k-1} \sigma_{i, i+1}^{2}=\sum_{i=1}^{k-1}\left(\frac{\operatorname{Var}\left(f_{i, i+1}\right)}{n_{i}^{2} f_{i, i+1}^{2}}+\frac{\operatorname{Var}\left(f_{i+1, i}\right)}{n_{i+1}^{2} f_{i+1, i}^{2}}\right)
\end{aligned}
$$

. For the periodic dihedral $\mathrm{CV}$, we employ periodic imaging to ensure the closure of the thermodynamics cycle in conformational pulling from $0^{\circ}$ to $360^{\circ}$. The round-trip error $\left(\Delta A_{0^{\circ}, 360^{\circ}}\right)$ is equally distributed along the whole free energy profile. With such setting, the free energy at $0^{\circ}$ is exactly the same with that at $360^{\circ}$. We note that in our previous studies and the current work, the round-trip error is rather small and about $0.1 \mathrm{kcal} / \mathrm{mol} .{ }^{85}$ This small size of cycle closure error indicates well converged sampling in the nonequilibrium ensemble. The curve or surface plotted from the free energy differences is the free energy profile or the free energy landscape of base flipping.

In our previous work, we proposed a new convergence criterion for bidirectional nonequilibrium pulling. If the state-specified SD is obviously smaller than the phase space overlap, the convergence behavior is good. ${ }^{86}$ The overlap scalar $^{88}$ can be computed from the variance of free energy difference,

$$
\sigma_{i j}^{2}=\frac{1}{n}\left(\left(O_{i j}\right)^{-1}-2\right)
$$

. Here the equal sample size rule is used and the sample size $n=n_{i}=n_{j} \cdot{ }^{43}$ Then we can write our criterion as

$$
\sigma_{i j}<O_{i j}
$$

. Note that in the above equation all quantities are dimensionless. We will discuss the validity of this criterion in the current nonequilibrium stratification in the base flipping case.

Brief review of the equilibrium umbrella sampling technique and reweighting schemes. The equilibrium umbrella sampling adds biasing potentials (often harmonic) to enhance the sampling in specific regions of the CV space. ${ }^{89}$ The definition of the biasing potential is similar to Eq. (1), and the difference is that the center of the biasing potential $\xi_{0}$ is no longer a function of time but remains constant during the simulation. Then, the statistics obtained from the biased ensemble are often reweighted with maximum likelihood estimators such as WHAM ${ }^{69-70}$ and $\mathrm{vFEP}^{71}$ to construct the free energy landscape. These two reweighting estimators often give similar results and the vFEP result is smoother due to the interpolation procedure employed to smooth the free energy profile.

\section{Computational Details}

\section{System preparation.}

The test system used to illustrate the applicability of our nonequilibrium stratification method is the model poly 
$\mathrm{A}_{7}-\mathrm{T}_{7}$ DNA duplex. The $\mathrm{A}_{7}-\mathrm{T}_{7}$ duplex is built with the $\mathrm{NAB}^{90}$ program and the system is described with the OL15 $5^{39-41}$ combination of modifications of AMBER force field. The system is solvated in TIP3P ${ }^{91-92}$ water molecules in a truncated octahedral box replicated in the whole space with periodic boundary conditions. Sodium ions parameterized for TIP3P water are added for neutralization. ${ }^{93-94}$ The minimum distance between any atoms originally present in solute and the edge of the periodic box is set to be $12 \AA$, and the resulted number of water molecules is 3275 .

\section{MD simulation.}

Firstly, we perform umbrella sampling to get the reference PMF. The umbrella windows are equally spaced from $0^{\circ}$ to $360^{\circ}$ with $5^{\circ}$ increments and the force constant of $100 \mathrm{kcal} / \mathrm{mol} \cdot \mathrm{rad}^{2}$ is used to ensure phase space overlap and enhance the sampling efficiency. This $5^{\circ}$ increments' spacing regime is widely used in base flipping studies. ${ }^{49-50,56}$ In each umbrella window, we perform 5000 cycles energy-minimization, $100 \mathrm{ps}$ NVT heating from $0 \mathrm{~K}$ to $300 \mathrm{~K}$, and 1 ns NPT equilibration before production runs. Then we perform $8 \mathrm{~ns}$ production sampling with the sampling interval of 2 ps. The quality of window spacing is checked with the overlap matrix, ${ }^{95}$ which is a quantitative estimator of phase space overlap. For reliable reweighting with perturbation based estimators, the main diagonal and its neighbors in the matrix should be appreciably larger than $0.03 .{ }^{95}$ In our case, the matrix is given in Fig. S1, from which satisfactory overlap can be seen.

Then we start our nonequilibrium stratification procedure. The segments are equally spaced from $0^{\circ}$ to $360^{\circ}$ with $2^{\circ}$ increments and the large force constants of $2000 \mathrm{kcal} / \mathrm{mol} \cdot \mathrm{rad}^{2}$ is used in nonequilibrium pulling and initial configuration sampling in order to achieve the stiff spring limit. In the initial configurational sampling procedure, in each configurational state, we perform 5000 cycles energy-minimization with steepest descent algorithm. The system is gradually heated from $0 \mathrm{~K}$ to $300 \mathrm{~K}$ in a NVT ensemble over a period of $100 \mathrm{ps}$, after which $2 \mathrm{~ns}$ NPT equilibration is performed. The initial configuration sampling is performed with the sampling interval of 2 ps. From our previous experience, the autocorrelation of the flipping dihedral (the $\mathrm{CV}$ used to describe the base flipping) typically decays to zero at this time. We calculate the statistical inefficiency and then subsample independent configurations to ensure all initial configurations are statistically uncorrelated. ${ }^{95}$ The nonequilibrium trajectories are then initiated from these uncorrelated configurations. For convergence check on the pulling speeds in nonequilibrium pulling, we tested a series of pulling speeds including 0.5 ps per $2^{\circ}$ segment, 1 ps/segment and $2 \mathrm{ps} / \mathrm{segment}$.

In all simulations, the $\mathrm{SHAKE}^{96}$ algorithm is applied to perform bond length constraints for bonds involving hydrogen atoms in all molecules. ${ }^{97}$ Langevin dynamics ${ }^{98}$ with the collision frequency of $5 \mathrm{ps}^{-1}$ are implemented for temperature regulation. Isotropic position scaling along with Berendsen barostat is employed to regulate the pressure. The time step used is $1 \mathrm{fs}$. A cutoff of 9 angstroms for non-bonded interactions in the real space is applied and long range electrostatics are treated with PME method. ${ }^{99}$ MD simulations are performed with AMBER ${ }^{100}$ suite and all other 
analysis are obtained with homemade codes.

\section{Result and discussion}

The minimum sampling time for converged PMF estimation from equilibrium umbrella sampling in base

flipping. The first question we need to answer is the minimum length of sampling time required for convergence. Firstly, we check the results from equilibrium umbrella sampling to provide a reference free energy profile for later comparison with the results from nonequilibrium stratification.

The block averaging method is often used to check the convergence of sampling in PMF construction. The question that how many samples are required for convergence or how long the simulation should be for converged sampling in base flipping simulations remains unanswered. The simulation time in convergence check differs among researches. For instance, some researchers just check the time-dependence of PMF for blocks of several hundred ps length ${ }^{50,59}$ while others tend to simulate at least several ns for each block. ${ }^{49}, 56$ When there are minor differences between blocks, the convergence is reached. In the current model poly A-T system, we revisit the length of block issue and emphasize the importance of the size of each block.

The free energy profiles obtained from different time blocks are presented in Fig. 2. We use 0.4 ns, 1 ns and 2 ns time blocks as these are the typical length of time blocks used in base flipping studies. The reason we compare the vFEP free energy curves is that $\mathrm{vFEP}$ uses cubic spline fitting and the free energy profiles are very smooth and easy to compare. In Fig. 2a, we show the free energy profiles calculated from 0.4 ns time blocks. We notice that the results from different 0.4 ns blocks show significant differences. The differences exist mostly in the flipping-out region. The height of the free energy barrier varies from $17.6 \mathrm{kcal} / \mathrm{mol}$ to $23.0 \mathrm{kcal} / \mathrm{mol}$. Such significant difference in free energy barrier (about $5 \mathrm{kcal} / \mathrm{mol}$ ) is much larger than the thermal energy $\mathrm{k}_{\mathrm{B}} \mathrm{T}$, and thus the sampling cannot be converged. By contrast, the inner base-paired state is relatively well sampled. The curvatures of free energy profiles calculated from different time blocks are very similar in this region. When we lengthen the time blocks to 1 ns, as is shown in Fig. 2b, we notice that most curves overlap in both the base-paired region and the flipping-out region, although there are still two lines deviating from the other. The PMF from first $1 \mathrm{~ns}$ sampling is obviously higher others and the 3-4 ns one is significantly lower than the other. The PMFs computed from the other 6 time blocks are almost identical. The reason for the deviation in the first $1 \mathrm{~ns}$ could be the nonequilibrium condition, while the 3-4 ns deviation should be caused by the non-ergodicity in the block, as all its neighbors are equilibrated. When the time blocks are further lengthened to $2 \mathrm{~ns}$, as is shown in Fig. 2c, we notice that the free energy profiles obtained from all time blocks are quite similar. Here as we are comparing the efficiency of equilibrium umbrella sampling and nonequilibrium stratification, we should neither underestimate the performance of the equilibrium umbrella sampling nor exaggerate the performance of 
the nonequilibrium method. Therefore, according to the above observation, we define $1 \mathrm{~ns}$ as the minimum length of time blocks for convergence. In practical use, to ensure the ergodicity, we recommend 2 ns time blocks for reliable estimation of the convergence behavior of the free energy profile in base flipping simulations.

As the vFEP PMF is smoothed by curve fitting, some details of the curve are missing or weaken. As in our nonequilibrium scheme we do not perform curve fitting and these details are reproduced, we then check the WHAM PMFs in Fig. 2d. We notice that the WHAM PMFs computed from different 1 ns blocks are also similar. Thus, in WHAM PMF construction we also use the $1 \mathrm{~ns}$ as the minimum length of time blocks for convergence.

Estimation of free energy landscape along base flipping pathway from nonequilibrium stratification. We then turn to the multi-stage nonequilibrium method proposed in the current work. Firstly, we check the statistical inefficiency in the initial configurational sampling in equilibrium states. As is shown in Fig. 3a, $\phi_{e q, i}$ are of similar values and minor statistical noises exist. As the sampling interval we use is 2 ps, the statistical inefficiency cannot be smaller than this value. We have tested smaller sampling intervals and the autocorrelation time of the flipping dihedral is similar. Therefore, we conclude that the typical sampling time required for each independent configuration in base flipping simulation is $2 \mathrm{ps}$.

Then we check the convergence of PMF on the pulling speed. The PMFs obtained with pulling speeds of $0.5 \mathrm{ps}$ per $2^{\circ}$ segment, 1 ps/segment and 2 ps/segment are shown in Fig. 3b, from which we notice that the pulling speed of $0.5 \mathrm{ps} / \mathrm{segment}$ is slow enough for converged PMF construction. Therefore, in the following parts we use the results obtained with this pulling speed as the results from nonequilibrium stratification.

The sample size dependence of the free energy landscape constructed with CE is given in Fig. 3c. The initial sample size is 5 and in each iteration 5 samples are added to the dataset. We notice that in the first several iterations there are still small changes in the free energy landscape. After 5 iterations, the PMF keeps unchanged and thus is fully converged. Therefore, the sample size required for convergence in nonequilibrium stratification is 25 .

Checking the time-dependence of the standard deviation (SD) in each state is also useful to determine convergence. According to Eq. (3), when the sample size becomes large, the standard deviation should decrease monotonically. Whether the $n_{i}^{-1}$ dependence is strictly followed can be used as a criterion for convergence. In the current base flipping case, the state-specified SDs shown in Fig. $3 \mathrm{~d}$ follow the $n_{i}^{-1}$ dependence and decrease monotonically after the $5^{\text {th }}$ iteration, while in the first several iterations there are fluctuations, indicating non-converged sampling. Therefore, according to the behavior of the SD profile, we can also determine the sample size required for convergence as 25 .

Another interesting phenomenon should be discussed is the relative size of the phase space overlap and the 
state-specified SD. In our previous multi-dimensional nonequilibrium pulling work, we pointed out that when SD is smaller than overlap scalar, the convergence behavior should be very well, and when SD is of similar size of overlap scalar, the convergence is acceptable. ${ }^{86}$ In the current nonequilibrium stratification in base flipping process, we also check whether this criterion is satisfied. As is shown in Fig. 4, with 5 samples the SD profile is of similar size with the overlap profile, and statistically significant noises are observed. When the sample size becomes 25 and 100, the SDs are much smaller than the overlap scalars, which is in consistent with our previous discussion about sample size required for convergence. Therefore, the $\sigma_{i j}<O_{i j}$ criterion can be used to check the convergence behavior of nonequilibrium stratification.

Practically, we recommend using all of the 3 criteria to check the convergence of nonequilibrium stratification. The first one is the time-invariant behavior of free energy landscapes. The second one is the $n^{-1}$ dependence of state-specified SD and the monotonically decreasing behavior. The third one is the $\sigma_{i j}<O_{i j}$ criterion.

Comparison between equilibrium umbrella sampling and nonequilibrium stratification. The free energy landscapes constructed from equilibrium umbrella sampling with WHAM and vFEP reweighting are compared with that obtained from our nonequilibrium stratification scheme in Fig. 5. From this plot, we notice that vFEP smooths out some details in the PMF while WHAM remains them. The PMF from nonequilibrium stratification agrees well with the WHAM PMF and the vFEP PMF. This agreement supports the applicability of nonequilibrium stratification on the construction of free energy profiles in base flipping.

Then we calculate the computational costs of the two enhanced sampling techniques and compare their efficiencies in PMF construction along the base flipping pathway. According to previous discussion, the sampling time required for convergence in equilibrium sampling is $1 \mathrm{~ns}$ in each umbrella window. According to the previous estimation of the typical autocorrelation time of the flipping dihedral, there are about 500 independent data points in each 1 ns sampling. In each 0.4 ns block, there are about 200 independent samples and such sample size seems a bit small for converged sampling in base flipping. There are 72 umbrella windows. The nonequilibrium stratification requires the pulling speed of $0.5 \mathrm{ps}$ per segment. As the pulling simulation is performed in both directions, the computational cost in each segment is multiplied by 2. 180 segments are divided and each segment requires 25 nonequilibrium realizations. The resulted statistics are summarized in Table 1, from which we notice that the nonequilibrium stratification is about 4-fold faster than umbrella sampling coupled with WHAM and vFEP. As neither the division of nonequilibrium segments nor the spacing of equilibrium umbrella windows is optimal, we would like to keep our conclusion modest. The nonequilibrium stratification scheme is of similar efficiency and accuracy with the equilibrium umbrella sampling scheme. 


\section{Conclusion}

In this work, we reported a general nonequilibrium stratification method for calculation of free energy landscapes of base flipping in duplex DNA. The free energy estimates are extracted from microscopic nonequilibrium works with the statistically optimal Crooks' Equation. The computational framework is easy to apply and gives comparable accuracy and efficiency compared with equilibrium umbrella sampling coupled with WHAM and vFEP. We further define the empirical simulation time required for converged PMF construction for umbrella sampling and nonequilibrium pulling. In equilibrium umbrella sampling, the empirical block length of $1 \mathrm{~ns}$ should be used for convergence check in PMF construction. We calculate the statistical inefficiency to dig the underlying reason for this phenomenon. It turns out that the typical autocorrelation time for the flipping dihedral is about 2 ps and thus each $1 \mathrm{~ns}$ block contain about 500 independent samples. The convergence criterion of $\sigma_{i j}<O_{i j}$, which is proposed previously in our multi-dimensional nonequilibrium pulling work, is tested here to determine the convergence of nonequilibrium stratification simulation in base flipping process. The convergence behavior determined by this criterion agrees with the $n^{-1}$ dependence of state-specified SD and the time-invariant behavior of free energy landscapes.

\section{Acknowledgement}

This work was supported China Scholarship Council and National Key R\&D Program of China (Grant no. 2016YFA0501700). Computer access to the CLAIX cluster of RWTH Aachen University and clusters of Forschungszentrum Juelich is gratefully acknowledged. We thank anonymous reviewers for valuable comments and critical reading.

\section{Conflicts of interest}

There are no conflicts of interest to declare. 


\section{References}

1. Zhang, Z.-L.; Wu, Y.-Y.; Xi, K.; Sang, J.-P.; Tan, Z.-J., Divalent ion-mediated DNA-DNA interactions: a comparative study of triplex and duplex. Biophys. J. 2017, 113 (3), 517-528.

2. Kadtsyn, E. D.; Anikeenko, A. V.; Medvedev, N. N., Molecular dynamics simulation of a DNA duplex labeled with triarylmethyl spin radicals. J. Mol. Liq. 2016, 221, 489-496.

3. Yin, Y.; Yang, L.; Zheng, G.; Gu, C.; Yi, C.; He, C.; Gao, Y. Q.; Zhao, X. S., Dynamics of spontaneous flipping of a mismatched base in DNA duplex. Proc. Natl. Acad. Sci. U.S.A. 2014, 111 (22), 8043-8.

4. Banavali, N. K., Partial Base Flipping Is Sufficient for Strand Slippage near DNA Duplex Termini. J. Am. Chem. Soc. 2013, 135 (22), 8274-82.

5. Leijon, M.; Gräslund, A., Effects of sequence and length on imino proton exchange and base pair opening kinetics in DNA oligonucleotide duplexes. Nucleic acids research 1992, 20 (20), 5339-5343.

6. O'Gara, M.; Klimaŝauskas, S.; Roberts, R. J.; Cheng, X., Enzymatic C5-cytosine methylation of DNA: mechanistic implications of new crystal structures forHhal methyltransferase-DNA-AdoHcy complexes. Journal of molecular biology 1996, 261 (5), 634-645.

7. Allan, B. W.; Reich, N. O.; Beechem, J. M., Measurement of the absolute temporal coupling between DNA binding and base flipping. Biochemistry 1999, 38 (17), 5308-5314.

8. Cal, S.; Connolly, B. A., DNA Distortion and Base Flipping by the EcoRV DNA Methyltransferase A STUDY USING INTERFERENCE AT dA AND T BASES AND MODIFIED DEOXYNUCLEOSIDES. J. Biol. Chem. 1997, 272 (1), 490-496.

9. O'Gara, M.; Roberts, R. J.; Cheng, X., A Structural Basis for the Preferential Binding of Hemimethylated DNA byHhal DNA Methyltransferase. Journal of molecular biology 1996, 263 (4), 597-606.

10. Wildes, A.; Theodorakopoulos, N.; Valle-Orero, J.; Cuesta-López, S.; Garden, J.-L.; Peyrard, M., Structural correlations and melting of B-DNA fibers. Physical Review E 2011, 83 (6), 061923.

11. Velizhanin, K. A.; Chien, C.-C.; Dubi, Y.; Zwolak, M., Driving denaturation: Nanoscale thermal transport as a probe of DNA melting. Physical Review E 2011, 83 (5), 050906.

12. Wong, K.; Pettitt, B., The pathway of oligomeric DNA melting investigated by molecular dynamics simulations. Biophys. J. 2008, 95 (12), 5618.

13. Blake, R.; Bizzaro, J., Jd; Day, G.; Delcourt, S.; Knowles, J.; Marx, K.; Santalucia, J. J., Statistical mechanical simulation of polymeric DNA melting with MELTSIM. Bioinformatics 1999, 15 (5), 370-375.

14. Gotoh, O., Prediction of melting profiles and local helix stability for sequenced DNA. Advances in biophysics 1983, 16, iii.

15. Lazurkin, Y. S.; Frank - Kamenetskii, M.; Trifonov, E., Perspectives report: Melting of DNA: Its study and application as a research method. Biopolymers 1970, 9 (11), 1253-1306.

16. Zimm, B. H., Theory of "melting"of the helical form in double chains of the DNA type. J. Chem. Phys. 1960, 33 (5), 1349-1356.

17. Travers, A., DNA dynamics: bubble 'n'flip for DNA cyclisation? Curr. Biol. 2005, 15 (10), R377-R379.

18. Zeida, A.; Machado, M. R.; Dans, P. D.; Pantano, S., Breathing, bubbling, and bending: DNA flexibility from multimicrosecond simulations. Physical Review E 2012, 86 (2), 021903.

19. Alexandrov, B. S.; Fukuyo, Y.; Lange, M.; Horikoshi, N.; Gelev, V.; Rasmussen, K. $\varnothing . ;$ Bishop, A. R.; Usheva, A., DNA breathing dynamics distinguish binding from nonbinding consensus sites for transcription factor YY1 in cells. Nucleic acids research 2012, 40 (20), 10116-10123.

20. Peyrard, M.; Cuesta-Lopez, S.; James, G., Nonlinear analysis of the dynamics of DNA breathing. Journal of biological physics 2009, 35 (1), 73.

21. Duduiala, C. I.; Wattis, J. A.; Dryden, I. L.; Laughton, C. A., Nonlinear breathing modes at a defect site in DNA. Phys Rev E Stat Nonlin Soft Matter Phys 2009, 80 (6 Pt 1), 061906.

22. Huang, N.; Banavali, N. K.; MacKerell, A. D., Protein-facilitated base flipping in DNA by cytosine-5-methyltransferase. Proceedings of the National Academy of Sciences 2003, 100 (1), 68-73.

23. Frank-Kamenetskii, M., How the double helix breathes. Nature 1987, 328 (6125), 17.

24. Pechlaner, M.; Donghi, D.; Zelenay, V.; Sigel, R. K., Protonation - Dependent Base Flipping at Neutral pH in the Catalytic Triad 
of a Self - Splicing Bacterial Group II Intron. Angew. Chem. Int. Ed. 2015, 54 (33), 9687-9690.

25. Englander, S. W.; Kallenbach, N. R., Hydrogen exchange and structural dynamics of proteins and nucleic acids. Quarterly reviews of biophysics 1983, 16 (4), 521-655.

26. Yakushevich, L. V., Is DNA a nonlinear dynamical system where solitary conformational waves are possible? J. Biosci. (Bangalore) 2001, 26 (3), 305-313.

27. Takeno, S.; Homma, S., Topological Solitons and Modulated Structure of Bases in DNA Double Helices: A Dynamic Plane Base-Rotator Model. Prog. Theor. Phys. 1983, 70 (1), 308-311.

28. Lifson, S.; Roig, A., On the theory of helix-coil transition in polypeptides. J. Chem. Phys. 1961, 34 (6), $1963-1974$.

29. Vedenov, A.; Dykhne, A. M.; Frank-Kamenetskiï, M., The helix-coil transition in DNA. Soviet Physics Uspekhi 1972, 14 (6), 715.

30. Dauxois, T.; Peyrard, M.; Bishop, A. R., Entropy-driven DNA denaturation. Physical Review E 1993, 47 (1), R44.

31. Muto, V.; Lomdahl, P.; Christiansen, P., Two-dimensional discrete model for DNA dynamics: longitudinal wave propagation and denaturation. Phys. Rev. A 1990, 42 (12), 7452.

32. Baird, N. C., Simulation of hydrogen bonding in biological systems: Ab initio calculations for NH3 NH3 and NH3 NH4+. Int. J. Quantum Chem 1974, 8 (S1), 49-54.

33. ZdravkoviĆ, S., Helicoidal Peyrard-Bishop Model of DNA Dynamics. Journal of Nonlinear Mathematical Physics 2011, 18 (sup2), 463-484.

34. Deng, M. L.; Zhu, W. Q., Stochastic dynamics and denaturation of thermalized DNA. Phys Rev E Stat Nonlin Soft Matter Phys 2008, 77 (2 Pt 1), 021918.

35. Kalosakas, G.; Rasmussen, K. Ø.; Bishop, A. R., Non-exponential decay of base-pair opening fluctuations in DNA. Chem. Phys. Lett. 2006, 432 (1-3), 291-295.

36. Joyeux, M.; Buyukdagli, S., Dynamical model based on finite stacking enthalpies for homogeneous and inhomogeneous DNA thermal denaturation. Phys Rev E Stat Nonlin Soft Matter Phys 2005, 72 (5 Pt 1), 051902.

37. Pérez, A.; Marchán, I.; Svozil, D.; Sponer, J.; Cheatham III, T. E.; Laughton, C. A.; Orozco, M., Refinement of the AMBER force field for nucleic acids: improving the description of $\alpha / \gamma$ conformers. Biophys. J. 2007, 92 (11), 3817-3829.

38. Ivani, I.; Dans, P. D.; Noy, A.; Pérez, A.; Faustino, I.; Hospital, A.; Walther, J.; Andrio, P.; Goñi, R.; Balaceanu, A., Parmbsc1: a refined force field for DNA simulations. Nat. Methods 2016, 13 (1), 55.

39. Zgarbová, M.; Šponer, J.; Otyepka, M.; lii, T. E. C.; Galindomurillo, R.; Jurečka, P., Refinement of the Sugar-Phosphate Backbone Torsion Beta for AMBER Force Fields Improves the Description of Z- and B-DNA. J. Chem. Theory Comput. 2015, 11 (12), 5723.

40. Zgarbová, M.; Luque, F. J.; Šponer, J. í.; Cheatham III, T. E.; Otyepka, M.; Jurecka, P., Toward improved description of DNA backbone: revisiting epsilon and zeta torsion force field parameters. J. Chem. Theory Comput. 2013, 9 (5), $2339-2354$.

41. Krepl, M.; Zgarbová, M.; Stadlbauer, P.; Otyepka, M.; Banáš, P.; Koca, J.; Cheatham III, T. E.; Jurecka, P.; Šponer, J. i., Reference simulations of noncanonical nucleic acids with different $\chi$ variants of the AMBER force field: quadruplex DNA, quadruplex RNA, and Z-DNA. J. Chem. Theory Comput. 2012, 8 (7), 2506-2520.

42. Wang, X.; Tu, X.; Zhang, J. Z. H.; Sun, Z., BAR-based optimum adaptive sampling regime for variance minimization in alchemical transformation: the nonequilibrium stratification. Phys. Chem. Chem. Phys. 2018, 20 (3), 2009-2021.

43. Wang, X.; Sun, Z., A theoretical interpretation of variance-based convergence criteria in perturbation-based theories. arXiv preprint arXiv:1803.03123 2018.

44. Sun, Z. X.; Wang, X. H.; Zhang, J. Z. H., BAR-based optimum adaptive sampling regime for variance minimization in alchemical transformation. Phys. Chem. Chem. Phys. 2017, 19 (23), 15005-15020.

45. Sun, Z.; Yan, Y. N.; Yang, M.; Zhang, J. Z., Interaction entropy for protein-protein binding. J. Chem. Phys. 2017, 146 (12), 124124.

46. Sun, Z.; Wang, X.; Song, J., Extensive Assessment of Various Computational Methods for Aspartate's pKa Shift. J. Chem. Inf. Model. 2017, 57 (7), 1621-1639.

47. Hooft, R. W.; van Eijck, B. P.; Kroon, J., An adaptive umbrella sampling procedure in conformational analysis using molecular dynamics and its application to glycol. J. Chem. Phys. 1992, 97 (9), 6690-6694. 
48. Mezei, M., Adaptive umbrella sampling: Self-consistent determination of the non-Boltzmann bias. J. Comput. Phys. 1987, 68 (1), 237-248.

49. Sun, Z.; Wang, X.; Zhang, J. Z. H., Protonation-dependent base flipping in the catalytic triad of a small RNA. Chem. Phys. Lett. 2017, 684.

50. Lemkul, J. A.; Savelyev, A.; MacKerell Jr, A. D., Induced polarization influences the fundamental forces in DNA base flipping. The journal of physical chemistry letters 2014, 5 (12), 2077-2083.

51. Dans, P. D.; Faustino, I.; Battistini, F.; Zakrzewska, K.; Lavery, R.; Orozco, M., Unraveling the sequence-dependent polymorphic behavior of $d(C p G)$ steps in B-DNA. Nucleic acids research 2014, 42 (18), 11304-11320.

52. Dans, P. D.; Ivani, I.; Hospital, A.; Portella, G.; González, C.; Orozco, M., How accurate are accurate force-fields for B-DNA? Nucleic Acids Research 2017, 45 (7), 4217.

53. Galindo-Murillo, R.; Robertson, J. C.; Zgarbová, M.; Sponer, J.; Otyepka, M.; Jurečka, P.; Cheatham III, T. E., Assessing the current state of AMBER force field modifications for DNA. J. Chem. Theory Comput. 2016, 12 (8), 4114-4127.

54. Dans, P. D.; Danilāne, L.; Ivani, I.; Dršata, T.; Lankaš, F.; Hospital, A.; Walther, J.; Pujagut, R. I.; Battistini, F.; Gelpí, J. L., Long-timescale dynamics of the Drew-Dickerson dodecamer. Nucleic acids research 2016, 44 (9), 4052-4066.

55. Hagan, M. F.; Dinner, A. R.; Chandler, D.; Chakraborty, A. K., Atomistic understanding of kinetic pathways for single base-pair binding and unbinding in DNA. Proc Natl Acad Sci U S A 2003, 100 (24), 13922-7.

56. Zheng, H.; Cai, Y.; Ding, S.; Tang, Y.; Kropachev, K.; Zhou, Y.; Wang, L.; Wang, S.; Geacintov, N. E.; Zhang, Y., Base flipping free energy profiles for damaged and undamaged DNA. Chem. Res. Toxicol. 2010, 23 (12), 1868-1870.

57. Qi, W.; Song, B.; Lei, X.; Wang, C.; Fang, H., DNA base pair hybridization and water-mediated metastable structures studied by molecular dynamics simulations. Biochemistry 2011, 50 (44), 9628-32.

58. Bianchi, C.; Zangi, R., Dual base-flipping of cytosines in a CpG dinucleotide sequence. Biophys. Chem. 1900, 188 (3), 14-22.

59. Banavali, N. K.; Jr, M. K., Free Energy and Structural Pathways of Base Flipping in a DNA GCGC Containing Sequence. Journal of Molecular Biology 2002, 319 (1), 141-160.

60. Keepers, J.; Kollman, P. A.; James, T. L., Molecular mechanical studies of base - pair opening in d (CGCGC): $d$ (GCGCG), dG5 d dC5, d (TATAT): d (ATATA), and dA5 - dT5 in the B and Z forms of DNA. Biopolymers: Original Research on Biomolecules 1984, 23 (11), 2499-2511.

61. Keepers, J. W.; Kollman, P. A.; Weiner, P. K.; James, T. L., Molecular mechanical studies of DNA flexibility: coupled backbone torsion angles and base-pair openings. Proceedings of the National Academy of Sciences 1982, 79 (18), 5537-5541.

62. Wang, Y.; Wang, Z.; Wang, Y.; Liu, T.; Zhang, W., The nearest neighbor and next nearest neighbor effects on the thermodynamic and kinetic properties of RNA base pair. J. Chem. Phys. 2018, 148 (4), 045101.

63. Wang, Y.; Gong, S.; Wang, Z.; Zhang, W., The thermodynamics and kinetics of a nucleotide base pair. J. Chem. Phys. 2016, 144 (11), 115101.

64. And, P. V.; Lavery, R., Base Flipping in DNA: Pathways and Energetics Studied with Molecular Dynamic Simulations. J. Am. Chem. Soc. 2002, 124 (25), 7272-7273.

65. Giudice, E.; Várnai, P.; Lavery, R., Base pair opening within B - DNA: free energy pathways for GC and AT pairs from umbrella sampling simulations. Nucleic acids research 2003, 31 (5), 1434-1443.

66. Giudice, E.; Várnai, P.; Lavery, R., Energetic and Conformational Aspects of A: T Base - Pair Opening within the DNA Double Helix. ChemPhysChem 2001, 2 (11), 673-677.

67. And, E. G.; Lavery, R., Nucleic Acid Base Pair Dynamics: The Impact of Sequence and Structure Using Free-Energy Calculations. J. Am. Chem. Soc. 2003, 125 (17), 4998.

68. Zwanzig, R. W., High temperature equation of state by a perturbation method. I.nonpolar Gases J.chem.phys 1954, 22 (8), 1420-1426.

69. Hub, J. S.; Groot, B. L. D.; Spoel, D. V. D., g_wham-A Free Weighted Histogram Analysis Implementation Including Robust Error and Autocorrelation Estimates. J. Chem. Phys. 2015, 6 (9), 3713-3720.

70. Ono, S.; Nakajima, N.; Higo, J.; Nakamura, H., The multicanonical weighted histogram analysis method for the free-energy landscape along structural transition paths. Chem. Phys. Lett. 1999, 312 (2-4), 247-254.

71. Lee, T. S.; Radak, B. K.; Pabis, A.; York, D. M., A New Maximum Likelihood Approach for Free Energy Profile Construction 
from Molecular Simulations. J Chem Theory Comput 2013, 9 (1), 153-164.

72. Priyakumar, U. D.; Mackerell, A. D., Base Flipping in a GCGC Containing DNA Dodecamer: A Comparative Study of the Performance of the Nucleic Acid Force Fields, CHARMM, AMBER, and BMS. J. Chem. Theory Comput. 2006, 2 (1), 187-200.

73. Sponer, J.; Bussi, G.; Krepl, M.; Banáš, P.; Bottaro, S.; Cunha, R. A.; Gil-Ley, A.; Pinamonti, G.; Poblete, S. n.; Jurečka, P., RNA structural dynamics as captured by molecular simulations: A comprehensive overview. Chem. Rev. 2018, 118 (8), $4177-4338$.

74. Park, H.; González, A. I. L.; Yildirim, I.; Tran, T.; Lohman, J. R.; Fang, P.; Guo, M.; Disney, M. D., Crystallographic and computational analyses of AUUCU repeating RNA that causes spinocerebellar ataxia type 10 (SCA10). Biochemistry 2015, 54 (24), 3851-3859.

75. Liberman, J. A.; Suddala, K. C.; Aytenfisu, A.; Chan, D.; Belashov, I. A.; Salim, M.; Mathews, D. H.; Spitale, R. C.; Walter, N. G.; Wedekind, J. E., Structural analysis of a class III preQ1 riboswitch reveals an aptamer distant from a ribosome-binding site regulated by fast dynamics. Proceedings of the National Academy of Sciences 2015, 112 (27), E3485-E3494.

76. Allen, W. J.; Wiley, M. R.; Myles, K. M.; Adelman, Z. N.; Bevan, D. R., Steered molecular dynamics identifies critical residues of the Nodamura virus B2 suppressor of RNAi. J. Mol. Model. 2014, 20 (3), 2092.

77. Jarzynski, C., A nonequilibrium equality for free energy differences. Phys. Rev. Lett. 1997, 78 (14), $2690-2693$.

78. Mallick, K.; Moshe, M.; Orland, H., Supersymmetry and Nonequilibrium Work Relations. Physics 2008.

79. Colizzi, F.; Bussi, G., RNA unwinding from reweighted pulling simulations. J. Am. Chem. Soc. 2012, 134 (11), $5173-5179$.

80. Do, T. N.; Carloni, P.; Varani, G.; Bussi, G., RNA/Peptide Binding Driven by Electrostatics Insight from Bidirectional Pulling Simulations. J. Chem. Theory Comput. 2013, 9 (3), 1720-1730.

81. Hummer, G.; Szabo, A., From the Cover: Free energy reconstruction from nonequilibrium single-molecule pulling experiments. Proceedings of the National Academy of Science 2001, 98 (7), 3658-3661.

82. Hummer, G.; Szabo, A., Free energy reconstruction from nonequilibrium single-molecule pulling experiments. Proceedings of the National Academy of Sciences 2001, 98 (7), 3658-3661.

83. Hummer, G.; Szabo, A., Free energy surfaces from single-molecule force spectroscopy. Cheminform 2005, 36 (39), $504-13$.

84. Paramore, S.; Ayton, G. S.; Voth, G. A., Extending the fluctuation theorem to describe reaction coordinates. J. Chem. Phys. 2007, 126 (5), 992-992.

85. Wang, X.; Tu, X.; Deng, B.; Zhang, J. Z. H.; Sun, Z., BAR-based Optimum Adaptive Steered MD for Configurational Sampling. https://chemrxiv.org/articles/BAR-based Optimum Adaptive Steered MD for Configurational Sampling/7322417 2018.

86. Wang, X.; He, Q.; Sun, Z., BAR-Based Multi-Dimensional Nonequilibrium Pulling for Indirect Construction of QM/MM Free Energy

Landscape. https://chemrxiv.org/articles/BAR-Based Multi-Dimensional Nonequilibrium Pulling for Indirect Construction of QM MM Fr ee Energy Landscape/7329176 2018.

87. Shirts, M. R.; Bair, E.; Hooker, G.; Pande, V. S., Equilibrium free energies from nonequilibrium measurements using maximum-likelihood methods. Phys Rev Lett 2003, 91 (14), 140601.

88. Bennett, C. H., Efficient estimation of free energy differences from Monte Carlo data. J. Comput. Phys. 1976, 22 (2), 245-268.

89. Kästner, J., Umbrella sampling. Wiley Interdisciplinary Reviews: Computational Molecular Science 2011, 1 (6), $932-942$.

90. Macke, T. J.; Case, D. A., Modeling unusual nucleic acid structures. American Chemical Society 1998, $379 \&$ ndash;393.

91. Jorgensen, W. L.; Chandrasekhar, J.; Madura, J. D.; Impey, R. W.; Klein, M. L., Comparison of Simple Potential Functions for Simulating Liquid Water. J. Chem. Phys. 1983, 79 (2), 926-935.

92. Price, D. J., A modified TIP3P water potential for simulation with Ewald summation. J. Chem. Phys. 2004, 121 (20), 10096-103.

93. Joung, I. S., Determination of alkali and halide monovalent ion parameters for use in explicitly solvated biomolecular simulations. J. Phys. Chem. B 2008, 112 (30), 9020-41.

94. Joung, I. S.; lii, T. E. C., Molecular Dynamics Simulations of the Dynamic and Energetic Properties of Alkali and Halide lons Using Water-Model-Specific Ion Parameters. J. Phys. Chem. B 2009, 113 (40), 13279-90.

95. Klimovich, P. V.; Shirts, M. R.; Mobley, D. L., Guidelines for the analysis of free energy calculations. J. Comput. Aided Mol. Des. 2015, 29 (5), 397-411. 
96. Ryckaert, J. P.; Ciccotti, G.; Berendsen, H. J. C., Numerical integration of the cartesian equations of motion of a system with constraints: molecular dynamics of $n$-alkanes. J. Comput. Phys. 1977, 23 (3), 327-341.

97. Miyamoto, S.; Kollman, P. A., Settle: An analytical version of the SHAKE and RATTLE algorithm for rigid water models. J. Comput. Chem. 1992, 13 (8), 952-962.

98. Pastor, R. W.; Brooks, B. R.; Szabo, A., An analysis of the accuracy of Langevin and molecular dynamics algorithms. Mol. Phys. 1988, 65 (6), 1409-1419.

99. York, D. M.; Darden, T. A.; Pedersen, L. G., The effect of long - range electrostatic interactions in simulations of macromolecular crystals: A comparison of the Ewald and truncated list methods. J. Chem. Phys. 1993, 99 (10), 8345-8348.

100. Case, D. A.; Cheatham, T. E.; Tom, D.; Holger, G.; Luo, R.; Merz, K. M.; Alexey, O.; Carlos, S.; Bing, W.; Woods, R. J., The Amber biomolecular simulation programs. J. Comput. Chem. 2005, 26 (16), 1668-88. 
Table 1. Total simulation time for converged PMF estimation in equilibrium umbrella sampling and nonequilibrium stratification. The total sampling time in nonequilibrium stratification is given by $N_{\text {segments }} * N_{\text {traj }} *\left(\phi_{\text {NEW }}+\phi_{\text {eq }}\right)$, where $N_{\text {segments }}$ denotes the number of segments and $N_{\text {traj }}$ represents the number of realizations per $2^{\circ}$ segment. In equilibrium umbrella sampling the time for nonequilibrium pulling $\phi_{\mathrm{NEW}}$ is zero and $5^{\circ}$ increments are used in window spacing. For convergence, the equilibrium umbrella sampling requires $1 \mathrm{~ns}$ sampling and thus 500 points in each window, while the nonequilibrium pulling requires 25 realizations in each segment.

\begin{tabular}{|c|c|c|c|c|c|}
\hline Method & $\begin{array}{c}\phi_{\mathrm{eq}} \text { for each } \\
\text { initial } \\
\text { configuration (ps) }\end{array}$ & $\begin{array}{c}\phi_{\mathrm{NEW}} \text { in } \\
\text { each } \\
\text { segment } \\
\text { (ps) }\end{array}$ & $\begin{array}{c}\text { Number of } \\
\text { segments }\end{array}$ & $\begin{array}{c}\text { Number of } \\
\text { realizations per } \\
\text { segment }\end{array}$ & $\begin{array}{l}\text { Total } \\
\text { simulation } \\
\text { time (ns) }\end{array}$ \\
\hline equilibrium umbrella sampling & 2 & 0 & 72 & 500 & 72.00 \\
\hline nonequilibrium stratification & $\begin{array}{l}\text { same with } \\
\text { equilibrium }\end{array}$ & $0.5 \times 2=1$ & 180 & 25 & 13.50 \\
\hline
\end{tabular}


Fig. 1. An illustration of the nonequilibrium stratification scheme used to calculate the free energy landscape of the base flipping process. We use $2^{\circ}$ increments in nonequilibrium stratification and bidirectional arrows represent bidirectional pulling and bidirectional reweighting of Crooks' Equation, which is used to recover the free energy differences between neighboring states from nonequilibrium work. According to the closure of the thermodynamic cycle, the free energy of $0^{\circ}$ and that of $360^{\circ}$ are exactly the same. At the center of the thermodynamic cycle, we present the definition of the reaction coordinate describing the base flipping process in the poly $\mathrm{A}_{7}-\mathrm{T}_{7}$ system. The pseudo-dihedral is defined by the four centers of mass. An illustrative picture of the flipping process is also given at the center of the thermodynamic cycle.

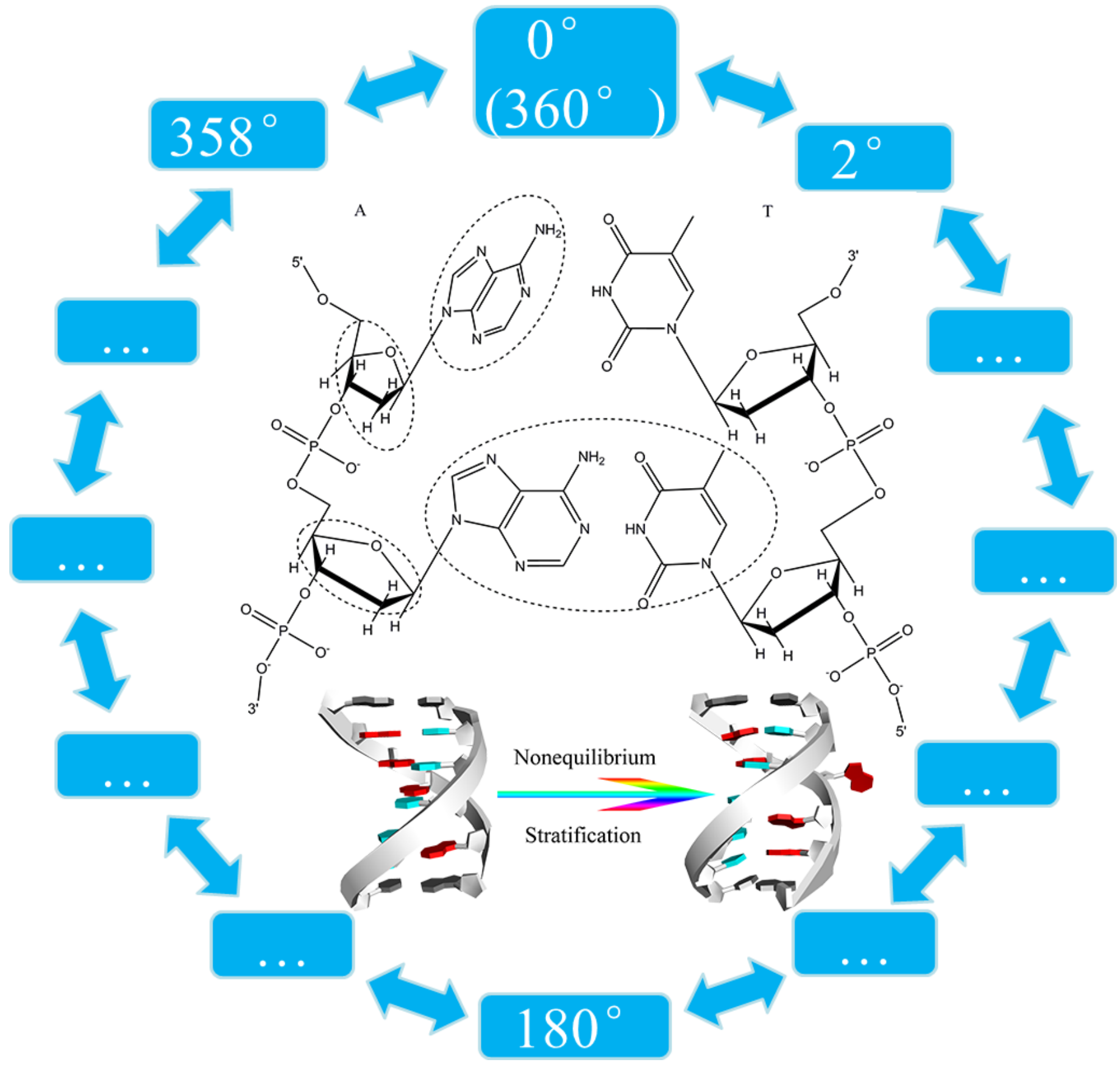


Fig. 2. The convergence behavior of free energy profiles constructed from equilibrium umbrella sampling simulations. a-c) Free energy profiles constructed with the vFEP method from $0.4 \mathrm{~ns}, 1 \mathrm{~ns}$ and 2 ns blocks, respectively. d) Free energy profiles constructed with the WHAM method from 1 ns blocks. The legend x-y ns represents the time blocks used to compute the free energy profiles.
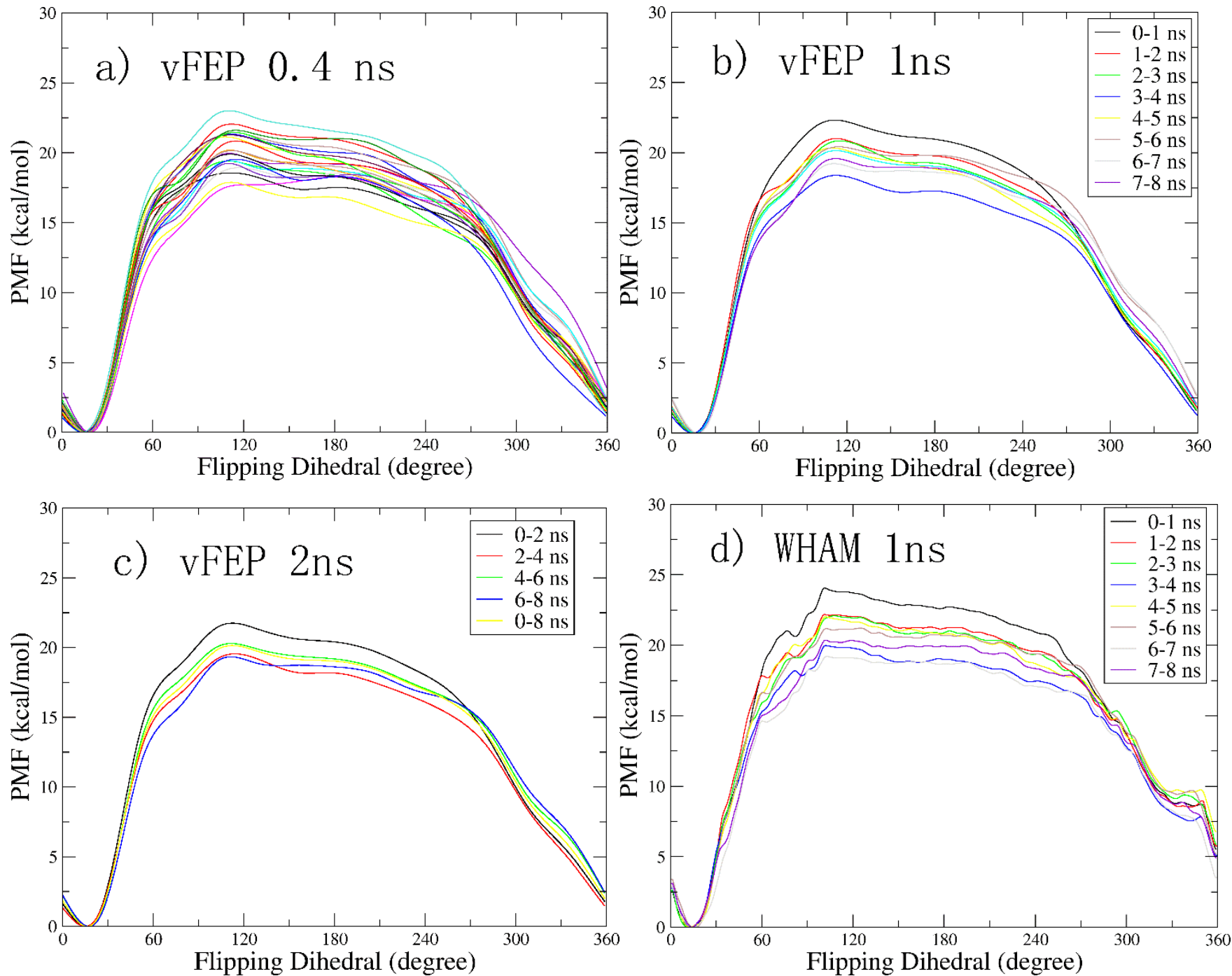
Fig. 3. a) the statistical inefficiency in each state in the initial configurational sampling and the convergence behavior of free energy profiles from nonequilibrium stratification on b) the pulling speed and c) the sample size. The legend $\mathrm{x}$ ps represents the pulling time for each $2^{\circ}$ segment. We notice that 0.5 ps per segment is slow enough for absolute convergence and thus the statistics under this pulling speed is used for discussion. The initial sample size is 5 and in each iteration further 5 samples are added to the dataset. There are small differences between the 5-sample PMF and the later ones. To avoid exaggerating the performance of nonequilibrium stratification, we use the sample size 25 as the sample size required for convergence. d) Time-evolution of state-specified SD.
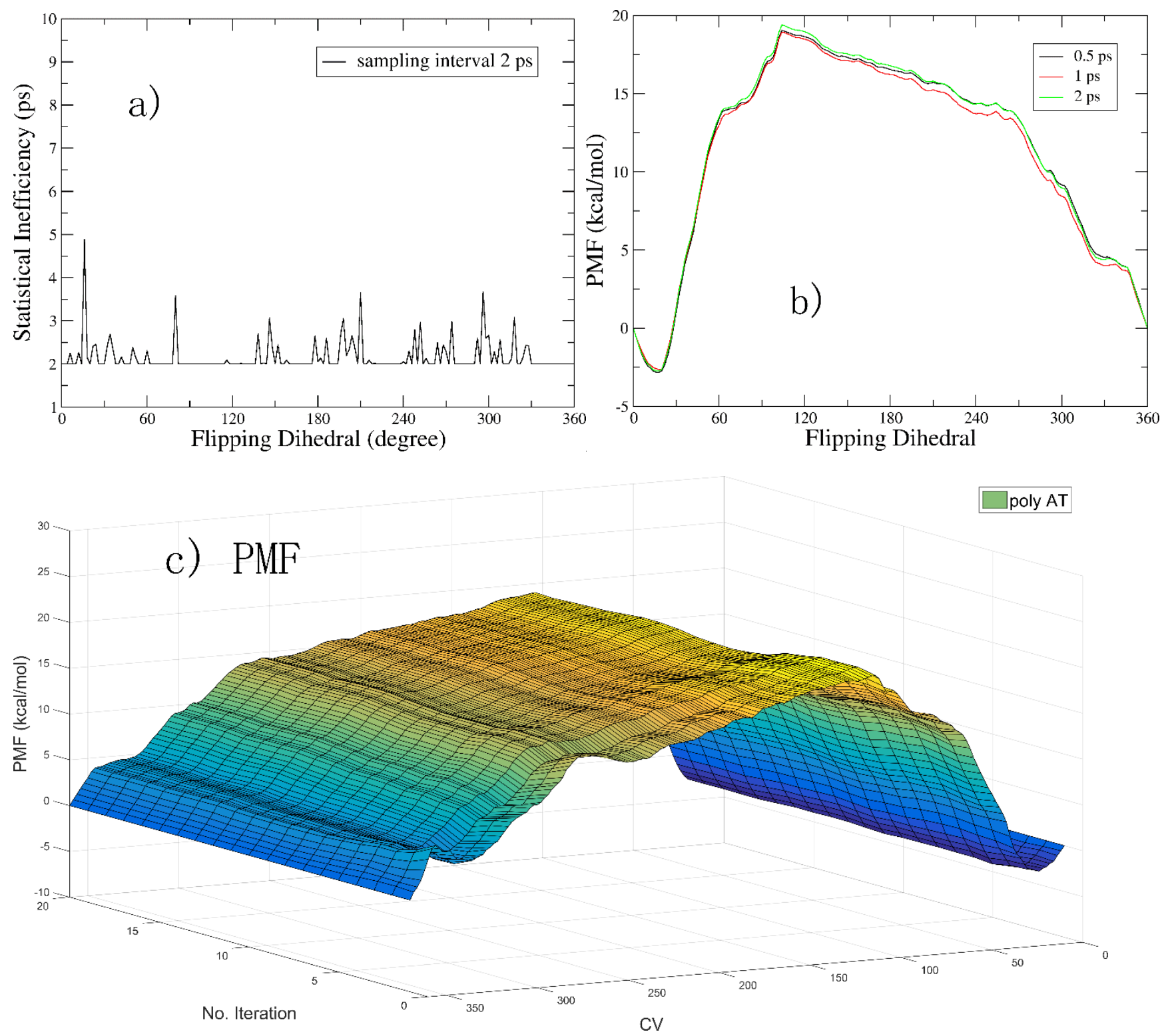
d) SD profile

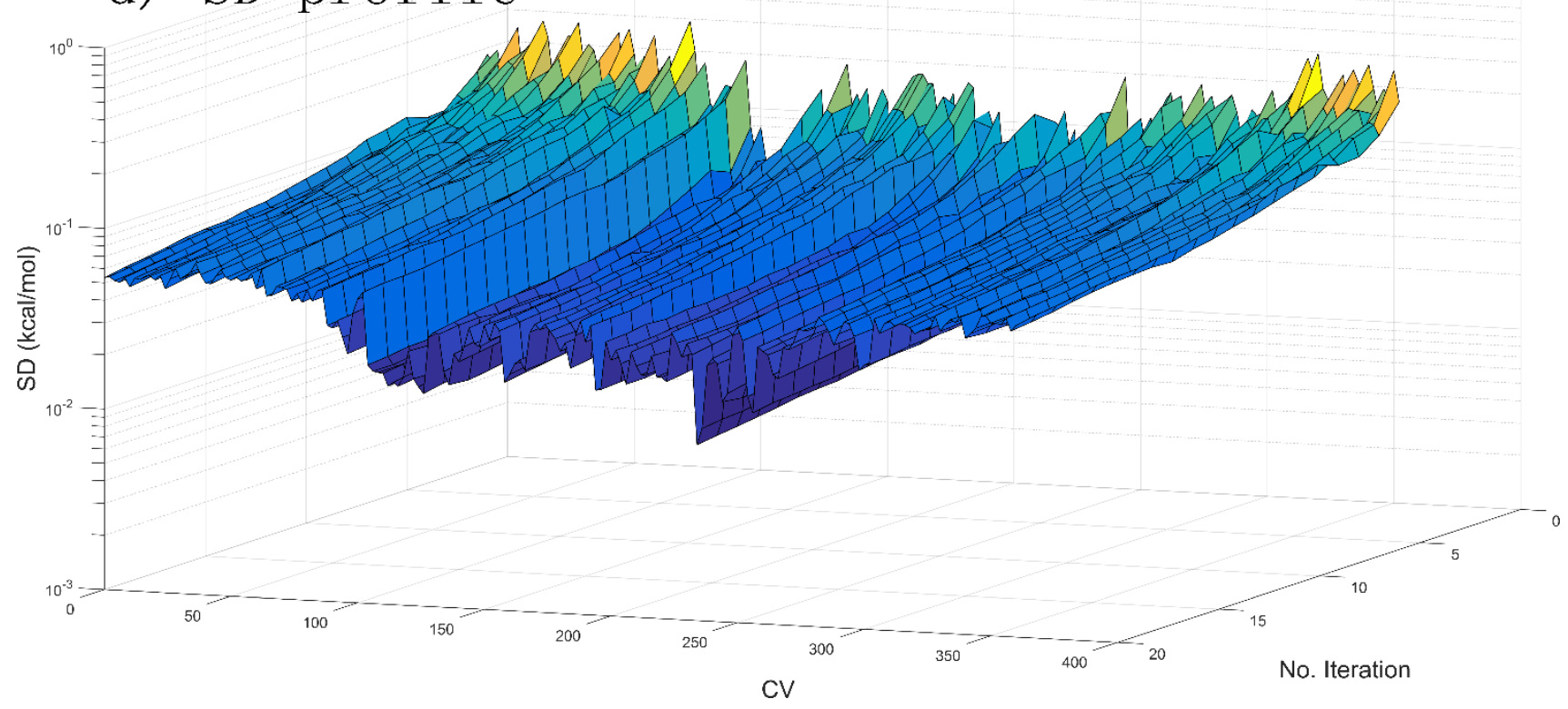


Fig. 4. Comparison between dimensionless SD profile and overlap profile in nonequilibrium stratification.

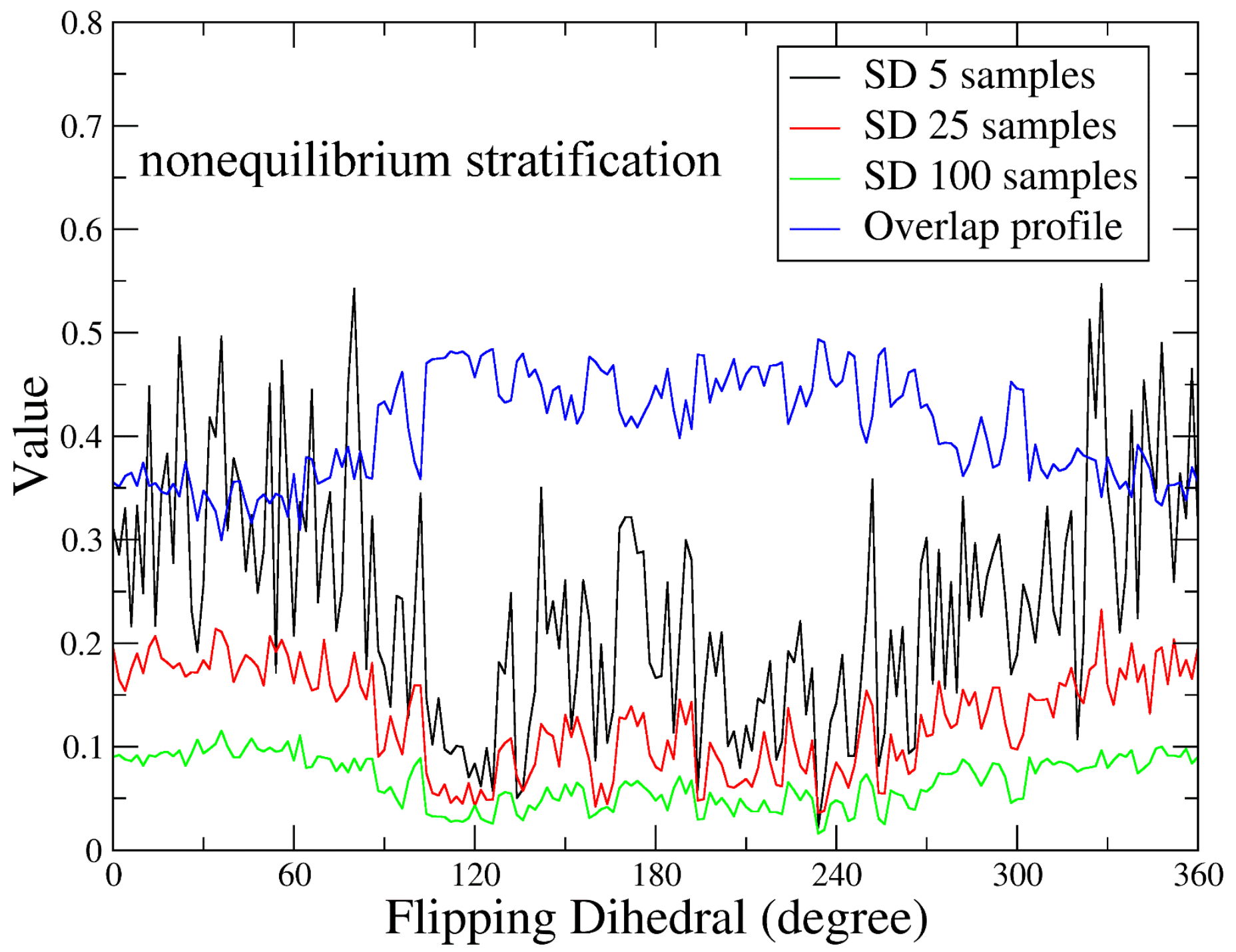


Fig. 5. Comparison between free energy landscapes obtained from equilibrium umbrella sampling and nonequilibrium stratification. In vFEP, as the curve fitting is used, the free energy of inner base-paired state is a bit overestimated, and the some details of PMF along the flipping pathway are smoothed out. The WHAM PMF remains these details. The PMF obtained from nonequilibrium work agrees with the equilibrium free energy profiles.

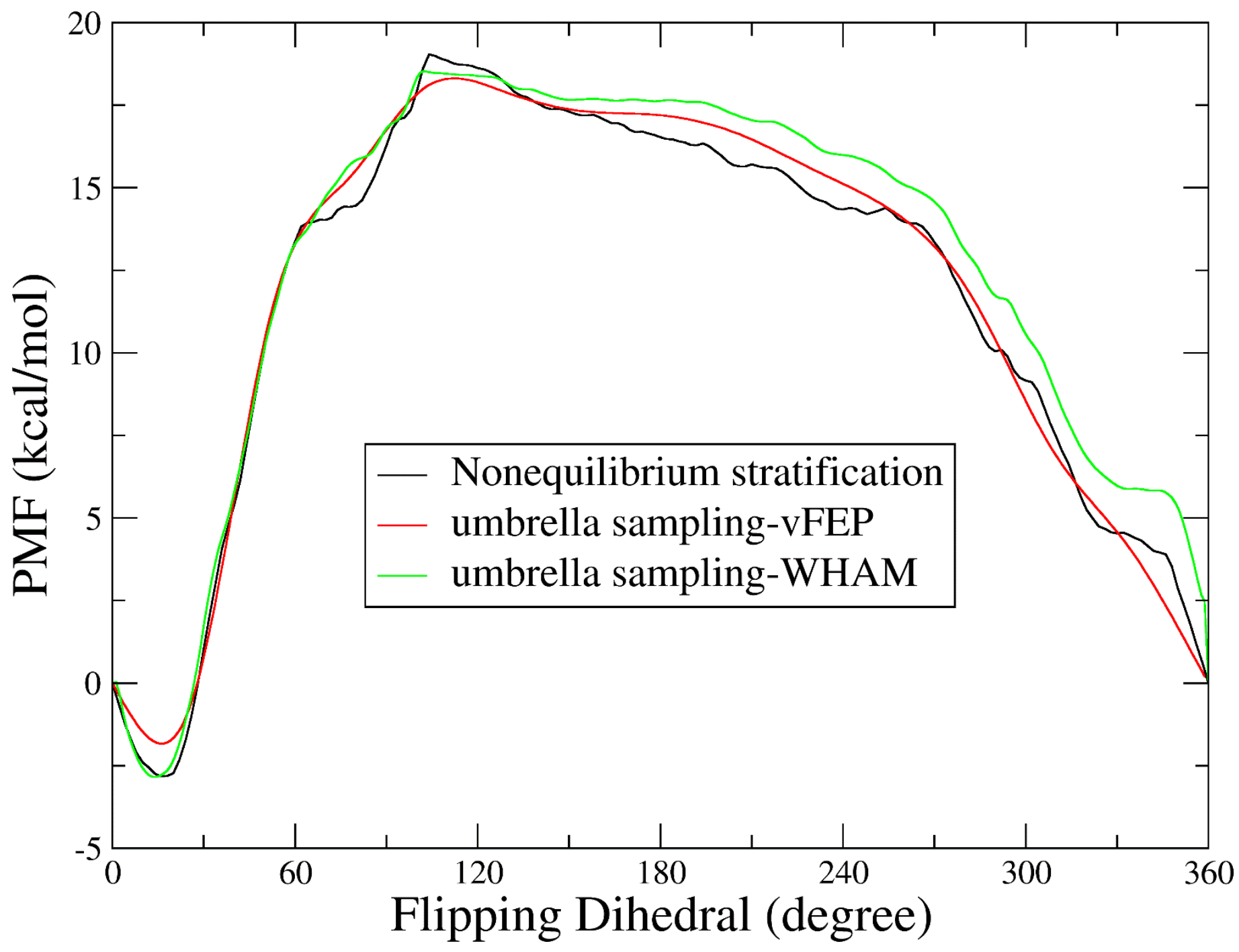




\title{
Supporting Information: Determination of Base Flipping Free Energy Landscapes from Nonequilibrium Stratification
}

\author{
Xiaohui Wang ${ }^{1,2}$ and Zhaoxi Sun ${ }^{1,3 *}$ \\ ${ }^{1}$ State Key Laboratory of Precision Spectroscopy, School of Chemistry and Molecular Engineering, East China \\ Normal University, Shanghai 200062, China \\ ${ }^{2}$ Institute of Computational Science, Università della Svizzera italiana (USI), Via Giuseppe Buffi 13, CH-6900, \\ Lugano, Ticino, Switzerland \\ ${ }^{3}$ Computational Biomedicine (IAS-5/INM-9), Forschungszentrum Jülich, Jülich 52425, Germany
}

*To whom correspondence should be addressed: z.sun@fz-juelich.de 
Fig. S1. Overlap matrix for phase space overlap check in equilibrium umbrella sampling.

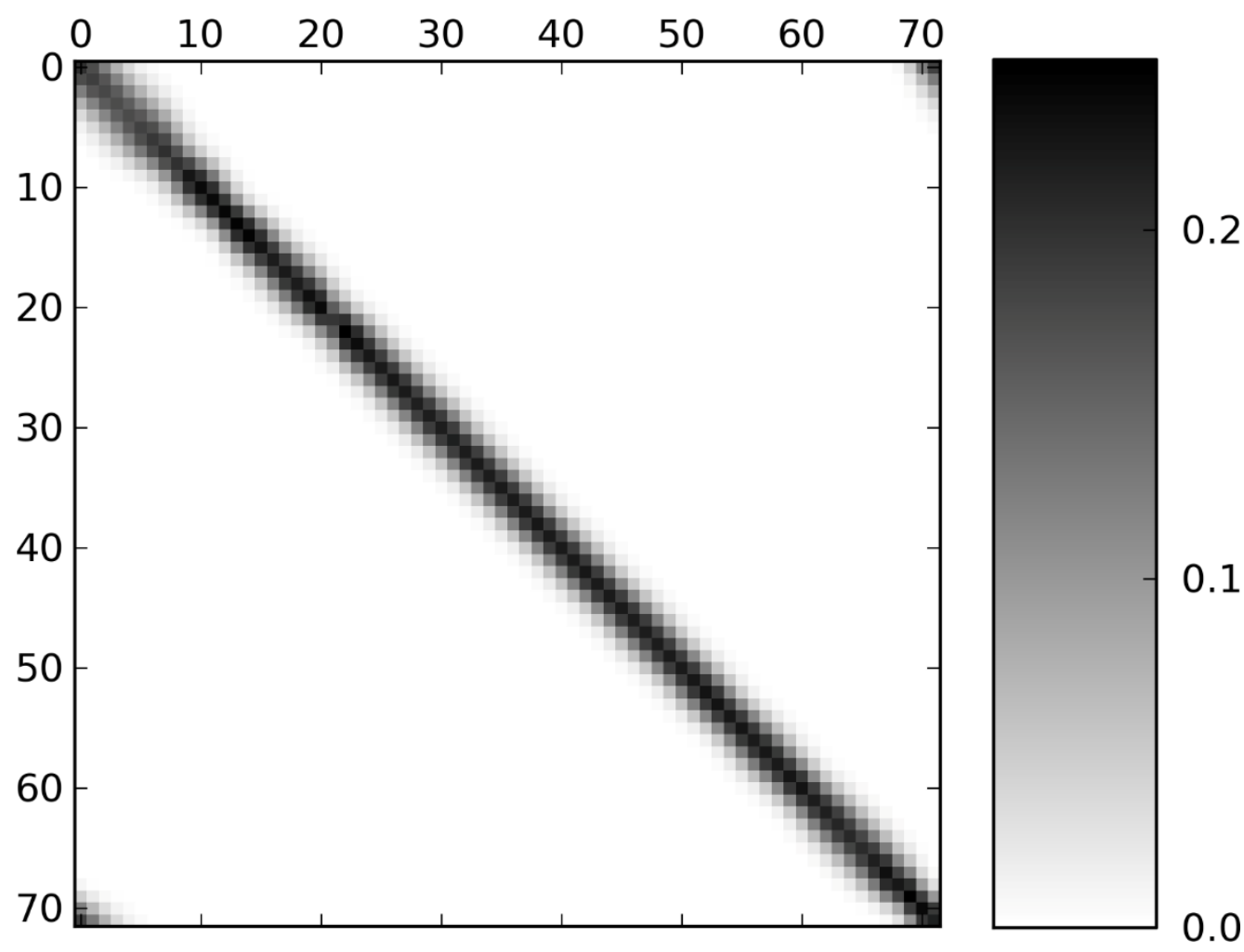

